\title{
Hooked! Modeling human disease in zebrafish
}

\author{
Cristina Santoriello' and Leonard I. Zon'1,2,3 \\ ${ }^{1}$ Department of Stem Cell and Regenerative Biology, Harvard University, Cambridge, Massachusetts, USA. \\ ${ }^{2}$ Stem Cell Program and Division of Hematology/Oncology, Children's Hospital Boston and Dana-Farber Cancer Institute, \\ Boston, Massachusetts, USA. ${ }^{3}$ Harvard Medical School, Boston, Massachusetts, USA.
}

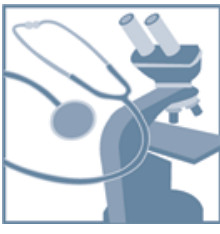

Zebrafish have been widely used as a model system for studying developmental processes, but
in the last decade, they have also emerged as a valuable system for modeling human disease.
The development and function of zebrafish organs are strikingly similar to those of humans,
and the ease of creating mutant or transgenic fish has facilitated the generation of disease
models. Here, we highlight the use of zebrafish for defining disease pathways and for discov-
ering new therapies.

\section{Introduction}

Danio rerio, better known as zebrafish, is a small vertebrate tropical water fish that has become one of the favored animal model systems for studying developmental processes and human disorders (Figure 1). Zebrafish share a high genetic similarity to humans, and approximately $70 \%$ of all human disease genes have functional homologs in zebrafish (1). Many advantages of zebrafish biology make it an attractive model for researchers: they have a high fecundity and can lay 200-300 eggs/week, the embryos are transparent and develop outside the body, making them particularly easy to study, and development is rapid, with major organs formed by 24 hours after fertilization. In addition, zebrafish are easy and inexpensive to raise and maintain, making it possible to keep thousands of animals in a laboratory at a reasonable cost. As a nonmammalian species, zebrafish do have certain disadvantages for modeling human disease; they lack some of the mammalian organs, such as lung and mammary gland, and phenotypic characteristics of diseases caused by orthologos genes can be very different in fish and human. In addition, the zebrafish genome includes many gene duplications, resulting in gene subfunctionalization and neofunctionalization (2).

Tools to model disease in zebrafish. Traditionally, zebrafish have been utilized as a forward genetic system. Chemical and insertional mutagenesis screens have led to the identification of a myriad of mutants with disruption of conserved genes that correlate to human disease loci (refs. 3, 4, and Figure 2). In addition, the DNA transposon system Sleeping Beanty - used in mouse for insertional somatic mutagenesis has been adopted in zebrafish and successfully used to identify conserved and novel cancer genes (5). More recently, the development of efficient gene knockdown technology has transformed zebrafish into a reverse genetic system. Morpholinos - antisense oligonucleotides that inhibit translation or affect splicing - can be used to transiently inhibit gene expression. Morpholinos are injected into embryos at the 1 - to 4-cell stage and remain active for several days $(6,7)$; since most of the organs have formed and are functioning within the first 5 days following fertilization, this technology permits a quick and easy probing of specific gene function in vivo (Figure 3). In contrast, this technology cannot be used to study gene function in mice because antisense oligonucleotides are rapidly diluted during mouse development.

In an effort to develop stable mutants, a technique called targetselected mutagenesis (TILLING) was developed $(8,9)$. This method

Conflict of interest: Leonard I. Zon is a founder and stockholder of Fate Inc. and a scientific advisor for Stemgent.

Citation for this article: J Clin Invest. 2012;122(7):2337-2343. doi:10.1172/JCI60434. combines standard ethylnitrosourea (ENU) mutagenesis with gene targeting in which genomic DNA from a large library of ENUmutagenized zebrafish is analyzed by exon sequencing to identify mutation(s) (Figure 3 and ref. 10). For example, this procedure generated missense mutations in the tumor suppressor p53 (11). A Zebrafish TILLING consortium (https://webapps.fhcrc.org/science/tilling/) has been established to facilitate the isolation of specific mutant lines. Similarly, with the development of new sequencing technologies, the Sanger Institute has developed the Zebrafish Mutation Project (http://www.sanger.ac.uk/Projects/D_rerio/zmp/). The ultimate goal of this project is to create an archive containing mutant alleles for all of the genes in the zebrafish genome, which are made available to the research community through the Zebrafish International Resource Center (ZIRC). To date, 4,469 mutant alleles are available.

Alternative methods to generate stable mutants have also been developed. Zinc finger endonucleases (ZFNs) are chimeric fusions between DNA-binding zinc finger proteins and the nonspecific cleavage domain of the FokI endonuclease. They can induce double-strand breaks in a specific genomic target sequence, which are imprecisely repaired by nonhomologous end joining (NHEJ) (12). ZFN mRNA is injected into one-cell-stage embryos to generate zebrafish carrying the desired genetic lesions. It has been shown that germ cell mosaicism in the identified ZFN allele-bearing founder fish is up to $50 \%$ (Figure 3 and ref. 13). The bottleneck of this approach is the generation of ZFNs with high and specific activity in vivo. The zebrafish community has designed a public database (ZFN Sequence Tag; pgfe. umassmed.edu/ZFNV1) to aid in the design and construction of ZFNs (14). ZFN-induced lesions have been reported in several genes including gata2, a zinc finger transcription factor that is essential for definitive hematopoiesis and that is associated with familial coronary artery disease (15). Its role in vasculature development is unknown, and the characterization of the ZNF mutant highlights a novel role for gata2 in artery morphogenesis (15). In a similar strategy, researchers have recently fused transcription activator-like (TAL) effectors to a FokI cleavage domain generating "TALENs" to disrupt target genes in zebrafish (16). These fusion proteins work very similarly to ZFNs, but are easier to design and assemble (17). It is not yet established whether TALENs induce off-target effects as have been shown for ZFNs (18).

To facilitate the over- and misexpression of genes of interest, germ line transgenesis has been greatly improved in zebrafish using several transposon-mediated systems $(19,20)$. Injection of these constructs into fertilized eggs can result in germ line transgenesis with $50 \%-80 \%$ efficiency. An expanding panel of transgenic zebrafish expressing fluorescent proteins in various cell types, organs, and anatomical 
A

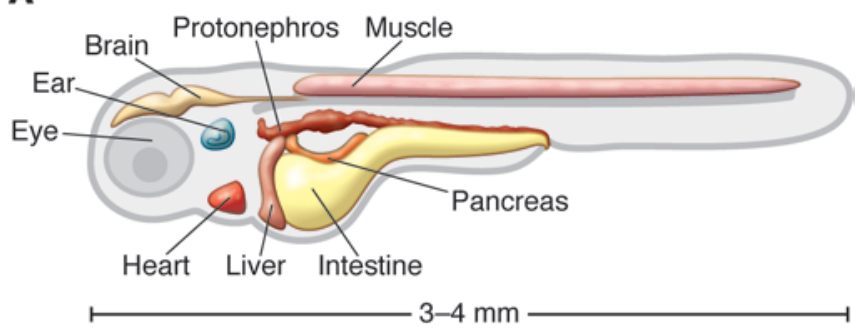

B

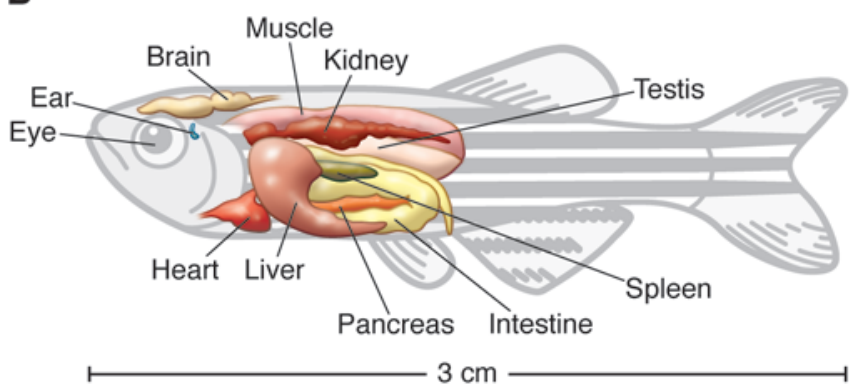

Figure 1

Drawing depicting zebrafish. Shown are larval (A) and adult (B) zebrafish organs. Larval age is 3 to 5 days post fertilization (dpf).

patterns has also been generated (21). Transgenic systems have been developed to provide precise temporal or spatial control of gene activation or inhibition, including inducible Cre-lox models, in which it is possible to control the temporal activity of the Cre recombinase by fusing it to a form of the estrogen receptor ligand-binding domain that has been modified to bind to tamoxifen $(20,22-24)$. The ease of making transgenic animals has led to many experiments probing overexpression of wild-type, constitutively active, or dominantnegative versions of a gene of interest. This has been useful for the generation of many disease models, including melanoma $(25,26)$.

Below, selected examples illustrate the distinct uses of zebrafish technology to create a model, explore disease biology, and to find new therapies.

\section{Hematological disorders}

The first zebrafish model of a human disease derived from positional cloning was established in 1998 (27). Isolated from a large forward genetic screen, the zebrafish mutant sauternes (sau) has a defect in hemoglobin production. The mutated gene encodes erythroid synthase $\delta$-aminolevulinate synthase (ALAS-2), which regulates the first step in heme biosynthesis; inactivation of this gene leads to congenital sideroblastic anemia in zebrafish and humans (27). Since then, several other mutants have been isolated from genetic screens that resemble human hematological diseases, including anemia (28-30), polycythemia (31), and porphyria $(4,32)$. The positional cloning of the hypochromic anemia mutant weissherbst (weh) identified ferroportin 1 as a novel iron transporter (28). The human ortholog was subsequently found mutated in patients affected by hemochromatosis, a disorder characterized by iron absorption defects (33). Other anemias have been phenocopied using morpholino-mediated knockdown $(34,35)$, including diamond blackfan anemia (DBA), which is modeled by knockdown of ribosomal protein RSP19 (36). Charac- terization of the RSP19 morphants and other ribosomal mutants revealed an activation of the $\mathrm{p} 53$ pathway, raising the possibility that a p53 family member could be targeted for DBA treatment $(34,37)$.

Acute lymphoblastic lenkemia. Acute lymphoblastic leukemia (ALL) is the most common type of childhood leukemia (38). The first transgenic cancer model established in zebrafish was a $\mathrm{T}$ cell leukemia model generated using a chimeric transgene encoding the mouse $c-M y c$ gene fused to GFP driven by a rag2 promoter (39). Within two months, injected zebrafish developed tumors in the thymus that spread to the gills, eye, abdominal organs, and muscle (39). Later, a double-transgenic line was developed carrying the lox-dsRED2-lox EGFP:mMyc and Cre driven by a heat shock promoter. After induction, these fish developed tumors with $81 \%$ penetrance (40). Another T-ALL leukemia model was established using an activated form of NOTCH1, which is mutated in over $50 \%$ of all human T-ALL cases. Zebrafish carrying the mutated NOTCH1 develop tumors with late onset, but when crossed to a line overexpressing bcl2, a more rapid tumor onset was observed, suggesting cooperation between the Notch pathway and bcl2-mediated apoptosis (41). bcl2 overexpression in both Notch- and Myc-induced T-ALL led to more aggressive tumors that were resistant to radiation (42). These studies demonstrate the utility of modifier screens in the identification of genetic interactions. These zebrafish T-ALL models are sensitive to the same chemotherapeutic drugs used in patients (43) and thus may be used in drug screens to identify novel therapeutics. In addition, a comparative study on copy number aberrations (CNAs) in zebrafish and humans revealed an overlap between T-ALL CNA genes across species, supporting zebrafish as a relevant model for studying human leukemias (44).

\section{Solid tumor models}

Fish and human solid tumors share a high degree of histological similarity. Chemical treatment, transplantation of mammalian cells, forward genetic screens, and reverse genetic approaches using knockouts and transgenes have been employed to generate zebrafish cancer models (reviewed in refs. 45, 46).

Melanoma. 50\%-60\% of melanoma tumor samples carry the activating mutation $B R A F^{V 600 E}$, which results in sustained activation of the BRAF/MEK1/2/ERK1/2 MAP kinase pathway (47). In addition to $B R A F$, another member of the MAP kinase pathway, the RAS oncogene (rat sarcoma viral oncogene homolog), is found mutated in approximately $20 \%$ of metastatic melanoma. In 2005 , Patton et al. generated the first zebrafish melanoma model, in which human $B R A F^{V 600 E}$ was expressed in melanocytes using the promoter of microphthalmia-associated transcription factor a (mitfa) (48). Fish injected with this mutant form of BRAF developed moles similar to human nevi, whereas injection of BRAF into $p 53^{M 214 K}$ mutant fish led to the formation of melanoma starting at four months of age. Although $p 53$ mutations are rare in human melanoma tumors, the gene is frequently functionally inactivated by loss of CDKN2A or other tumor suppressor pathways (49). More recently, a model of melanoma formation independent of p53 activity was developed using a UAS $\backslash$ GAL4 system. A cross between a driver line expressing the transactivator GAL4 driven by the $c$-kit (kit-a in fish) promoter and a responder line expressing the human activated form of H-RAS ${ }^{G 12 V}$ led to the generation of fish that display a hyperpigmented phenotype and the formation of tumors beginning at four weeks after fertilization (50).

Ceol et al. used the BRAFV600E/p53 $3^{\text {M214K }}$ fish model to develop a method for testing the role of genes in a recurrently amplified 


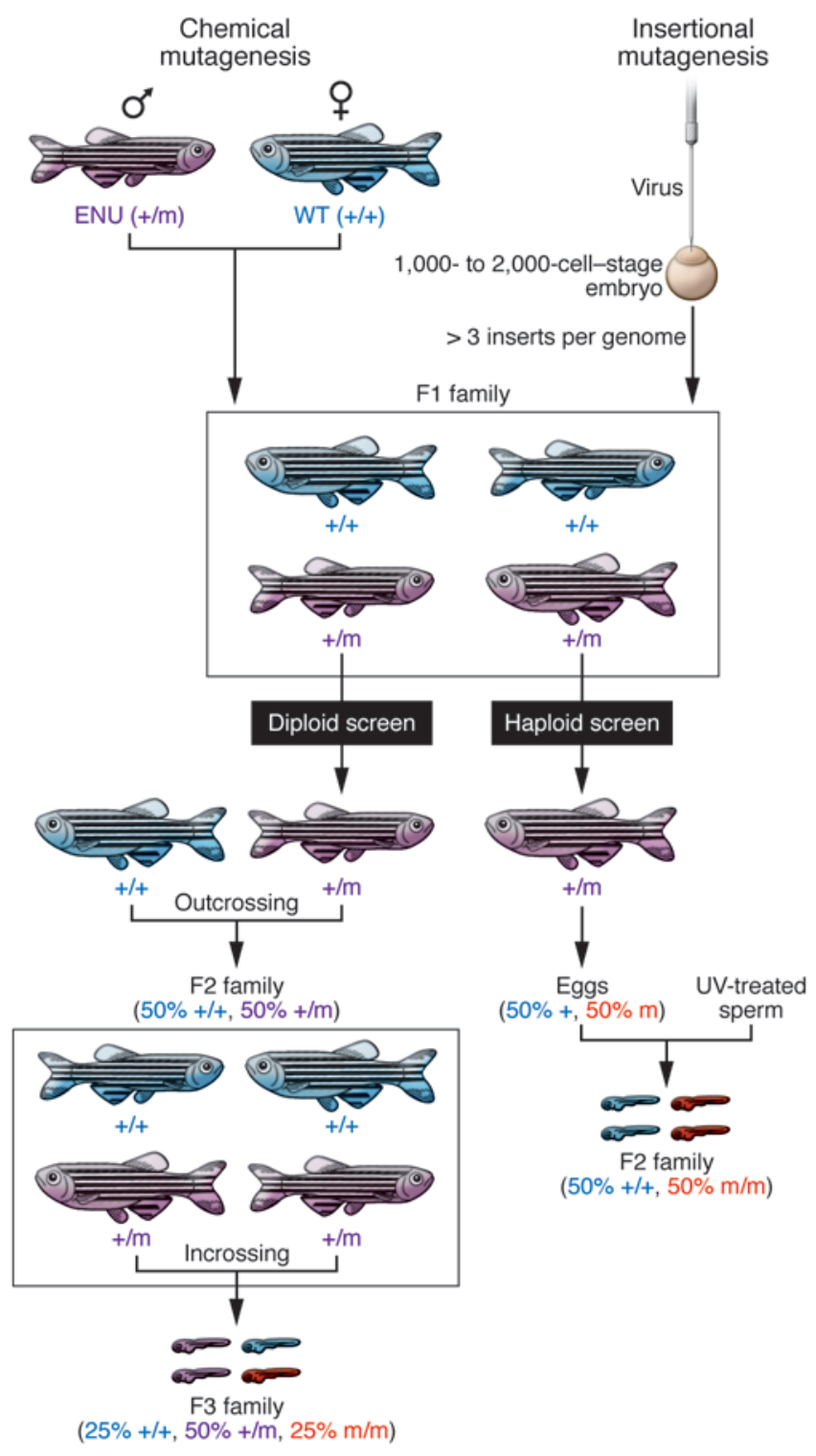

region of human chromosome 1 in melanoma. They found that $S E T D B 1$, a histone methyltransferase that is amplified in as many as $30 \%$ of human melanomas, accelerated tumor formation (51). These melanoma models recapitulate the pathology of the human melanoma and can be utilized as a cost-effective system for screening of anticancer compounds.

\section{Heart disorders}

Zebrafish are well suited for studying heart development and disorders because oxygen and nutrients can reach tissues by passive diffusion in embryos, so those with malformed hearts can still develop and survive for 2 to 3 days after fertilization. Forward genetic screens led to the identification of mutants with heart defects resembling human dilated cardiomyopathies (DCMs) (52) characterized by enlargement of the ventricle, atrium, or both and by decreased myocardial contractility. The silent heart and pickwick mutants are characterized by poor heart contractility, and cloning of the mutations led to the identification of cardiac troponin $\mathrm{T}$ (tnnt2) (53) and the large sarcomeric Titin protein (54), respectively. Mutation of TNNT2 in humans causes

\section{Figure 2}

Forward genetics approaches to generate zebrafish disease models. Chemical mutagenesis (left). Adult males that have been mutagenized by treatment with ENU are crossed to a wild-type female to create an F1 generation that contains a random set of point mutations in their genome. In a diploid-based screen, members of the $\mathrm{F} 1$ are outcrossed to wild type to increase the number of fish carrying specific recessive mutations. The F2 generation is subsequently intercrossed to generate F3 progeny, which can be analyzed phenotypically for recessive defects. One-fourth of the F2 family intercrosses will produce mutant progeny in one-fourth of the F3 embryos. In a haploid screen, eggs obtained from F1 females are fertilized with UV-treated sperm to generate haploid embryos. The haploid clutch derived from a heterozygous female $(+/ \mathrm{m})$ will contain $50 \%$ mutant and $50 \%$ wild-type embryos. Insertional mutagenesis (right). Virus is injected into 1,000- to 2,000-cell-stage embryos. F1 fish carrying more then 3 insertions are subsequently bred. The F2 generation is screened employing the same breeding scheme used for the chemical-based mutagenesis.

DCM and sudden death in young athletes. Similarly, laminin $\alpha-4$ and integrin-linked kinase mutations cause heart failure in zebrafish by affecting cardiomyocytes and endothelial cells, and mutations in these genes have been linked to familial DCM in humans (55).

Additional heart mutants, including reggae and breakdance, which display short QT syndrome and long QT syndrome, respectively, have been identified in mutagenesis screens (56). Both mutants carry mutations in the ether-à-go-go-related gene (zERG), the major subunit of a potassium channel $(57,58)$. Peal et al. performed a small molecule screen using the breakdance mutants and identified flurandrenolide and a novel compound 2-methoxy-N(4-methylphenyl) benzamide (2-MMB) as small molecules that can rescue the breakdance phenotype. Such screens may lead to novel therapeutics for human arrhythmia (58).

Myocardial infarction. Compared with humans, young mice (59) and fish (60) have faster ability to regenerate heart tissue. Myocardial infarction (MI) has been recently modeled in fish using cryoinjury, which results in massive cell death (61). To identify the source of the newly formed cardiomyocytes following this injury, Jopling et al. used a Cre/lox lineage tracing system and found that regenerated cardiomyocytes arise from differentiated cardiomyocytes that undergo limited dedifferentiation characterized by structural changes and expression of cell-cycle progression genes (62). These data suggest that zebrafish heart regeneration is driven by preexisting cardiomyocytes rather than by progenitors, as previously suggested (63). Thymosin b4, a small ubiquitous protein, appears to be one signal that triggers the formation of new cardiac tissue and blood vessels in fish and in mouse $(64,65)$. These observations raise the hope of finding new heart-repair drugs using zebrafish.

\section{Muscle disorders}

Duchenne muscular dystrophy. Duchenne muscular dystrophy (DMD) is a lethal genetic disorder characterized by wasting of muscle tissue caused by mutations in the dystrophin gene. Zebrafish strains with mutations in the dystrophin gene (called $d m d$ or sapje) were identified from a large forward genetic screen (66) and have a phenotype similar to the human disease, displaying progressive degeneration of skeletal muscle. Currently, there is no cure for DMD and the treatments employed so far are aimed at controlling symptoms to maximize quality of life. Zebrafish $d m d$ mutants have been used in a chemical suppressor screen to identify potential compounds that can correct the disease pathology, as detected by alterations in birefringence, which measures muscle integrity (67). This screen 
A

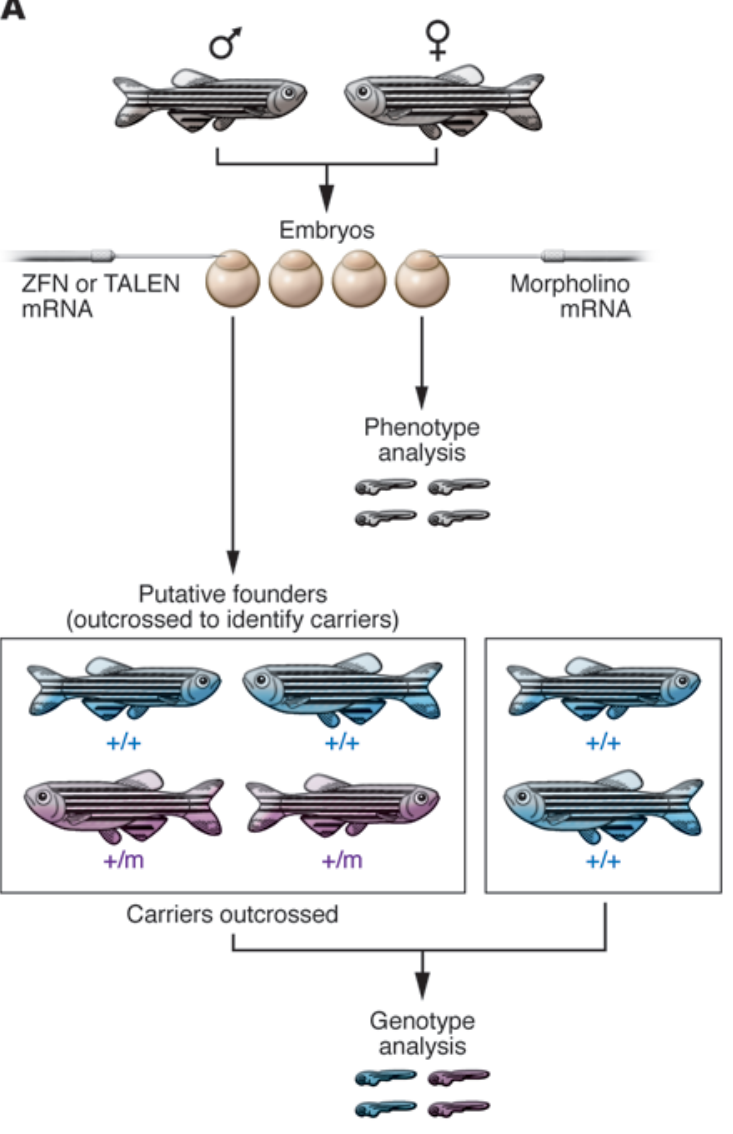

B

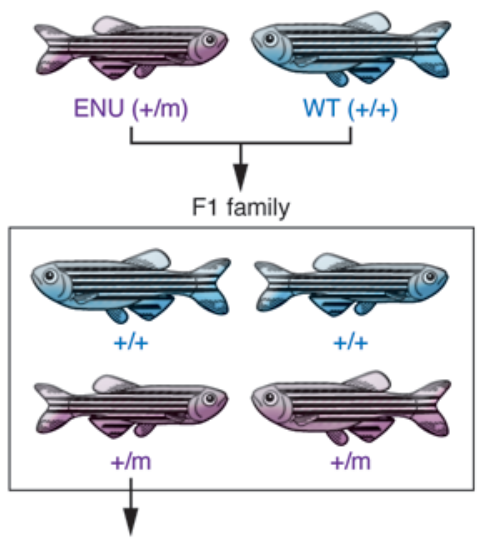

Tail clip or sperm DNA

PCR amplification of exons
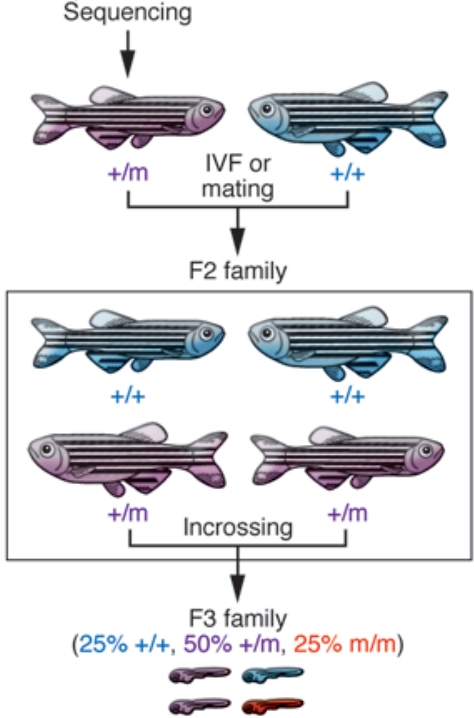

Figure 3

Reverse genetic approaches to generating zebrafish disease models. (A) Morpholinos or mRNA are injected into embryos to transiently knock down or overexpress a potential disease gene. Phenotypic analysis is performed on these embryos. Alternatively, mRNA encoding ZFN or TALEN is injected into the embryos. Putative founders are raised to adulthood and outcrossed to identify carriers. Founders carrying the allele of interest are then outcrossed to generate an F1 population. Heterozygous F1 carriers are identified by genotyping. (B) TILLING: adult males that have been mutagenized by treatment with ENU are crossed to wild-type females. A DNA library of tailfin clips or frozen sperm is prepared from F1 males. PCR amplification of exons of a specific gene of interest followed by sequencing is performed on the genomic library. Once a mutation is identified, the fish carrying the mutation will be recovered by crossing the corresponding fish from the live library to a wild-type fish or by thawing the cryopreserved sperm and using it for in vitro fertilization (IVF). The resulting progeny are then intercrossed to generate heterozygous and homozygous mutants for the disease gene.

revealed a number of compounds that appear to effectively reduce dystrophic symptoms in zebrafish. In particular, PDE5 inhibitors appear to be useful, and they have also been shown to be effective in the $m d x$ mouse model of muscular dystrophy (68).

\section{Kidney disorders}

Zebrafish have become a popular model for studying renal diseases thanks to the anatomical simplicity of their kidneys. Polycystic kidney disease (PKD), nephronophthisis, acute kidney injury (AKI), and a range of ciliopathies have been modeled in zebrafish (reviewed in ref. 69).

Several studies suggest that cilia (microtubule-based hair like organelles) play a central role in the etiology of PKD. The proper function of cilia prevents cystic formation and this hypothesis has been largely supported by the characterization of several zebrafish mutants carrying mutations in cilia proteins, such as intraflagellar transport proteins and LRRC50 (70-72).

Several ciliopathies including Bardet-Biedl syndrome (BBS), nephronophthisis (NPHP), Jeune, Joubert, oro-facial-digital (OFD1), and Meckel (MKS) syndromes have been modeled in zebrafish using morpholinos for ciliopathy candidate genes. The use of drugs such as rapamycin and roscovitine ameliorate the renal phenotype observed in these morphant embryos, suggesting that zebrafish can be used to identify potential therapeutic agents for renal cystitis (73).

The zebrafish kidney is also a valuable system for studying AKI because, as opposed to mammals, fish can generate new nephrons throughout their life and regenerate new nephrons after injury 
(74). Diep et al., in a series of transplantation experiments, were able to identify LIM homeobox 1a-positive (lhx1a-positive) cells as adult self-renewing nephron stem/progenitor cells (74). These findings pave the way for isolating similar cells in mammals with the aim of developing novel renal regenerative therapies.

\section{Central nervous system disorders}

Many studies have focused on the use of zebrafish as a model system for neurological, neurodegenerative, behavioral, and ocular disease, and these have been extensively reviewed elsewhere (75-78). Human neurodegenerative diseases including Parkinson, Huntington, and Alzheimer diseases have been successfully modeled in zebrafish, and the orthologs of major disease-associated genes have been identified in zebrafish (78).

In recent years, zebrafish have also been successfully used for behavioral screening of neuroactive drugs $(79,80)$. The search for new drugs to alleviate psychiatric and central nervous system disorders is a difficult task and, since in vitro studies cannot predict therapeutic outcomes in vivo, the use of whole-animal systems has become necessary. Kokel et al. used a stereotypical motor behavior called the photomotor response to screen 14,000 compounds in an automated fashion. Using phenotypic comparisons and computational techniques, the authors were able to identify new acetylcholinesterase and monoamine oxidase inhibitors (79). Rihel et al. screened nearly 4,000 compounds using an automated rest/wake behavioral assay. In this study, small molecules were clustered according to the behavioral phenotype observed. Many modulators of neurotransmitter systems, including the noradrenaline, serotonin, dopamine, GABA, glutamate, histamine, adenosine, and melatonin systems, induced sleep/wake phenotypes in zebrafish similar to those observed in mammals (80). This study suggests that factors including the potassium channel ERG are involved in controlling rest and locomotor activity and that modulation of these pathways could represent novel therapeutic strategies in the treatment of sleep disorders.

\section{Ocular disorders}

The zebrafish eye is similar in morphology, physiology, gene expression, and function to the human eye. Several zebrafish mutants displaying eye defects and visual impairment have been identified, revealing that signaling pathways including sonic hedgehog (Shh), nodal, and retinoic acid are involved in eye development and disease (81). Pharmacological intervention in these mutants has shed light on the mechanisms of these diseases. For example, the blowout mutant, which harbors a mutation in patched 1 (a negative regulator of Shh) revealed that pharmacologic inhibition of the Hedgehog pathway rescues the coloboma phenotype characterized by open choroid fissure (82). Rather surprisingly, lamb (laminin beta 1) and pax2 mutants displaying coloboma are rescued upon treatment with gentamicin and paromycin, two aminoglycoside drugs that most likely allow translational read-through of nonsense mutations (83). This work suggests a possible treatment for coloboma.

Neuhauss et al. used for the first time optokinetic and optomotor behavioral assays to analyze more than 400 mutants previously identified based on defects in organ formation, tissue patterning, and pigment formation. This study uncovered mutations that led to lens degeneration (bumper), melanin deficiency (sandy), lack of ganglion cells (lakritz), ipsilateral misrouting of axons (belladonna), and optic-nerve disorganization (grumpy and sleepy) (84). Several other ocular diseases, including glaucoma and retinal degenera- tion, have also been modeled in fish (76). Retinal degeneration is observed in several ciliopathies such as Leber's congenital amaurosis (LCA), BBS, Senior-Loken syndrome, Joubert syndrome, and MKS (85). The phenotype of the zebrafish mutants (oval, elipsa, fleer) closely resembles some of these human ciliopathies, including defects in photoreceptor outer segment formation. Positional cloning of the oval locus identified a mutation in the intraflagellar transport protein 88 (IFT88), which is a component of the IFT complex. This complex is involved in the generation and maintenance of ciliated structures (86). The further characterization of these mutants should help to clarify the roles of individual IFT particle members in the formation and survival of photoreceptor cilia.

\section{Future perspectives}

Due to their versatile and unique features, we believe zebrafish will play an increasingly prominent role in the identification and study of disease genes and therapeutic discovery.

Interfacing with buman genetics and genomics. Whole-genome sequencing and genome-association studies have identified candidate human disease genes. The zebrafish system is well poised for functionally characterizing such genes and can be used to examine coding and noncoding function in vivo. Several studies using genomic approaches have identified gene variants whose function in disease pathology has been successfully tested in zebrafish, including melanoma (87), cardiomyopathy (88), polyneuropathy (89), neurodegenerative disease $(90,91)$, and ciliopathies (92). Recently, Gieger et al. carried out a high-powered meta-analysis of genome-wide association studies (GWAS) in nearly 67,000 individuals to identify putative novel regulators of megakaryopoiesis and platelet formation (93). Out of the 68 genomic loci associated with platelet count and volume mapping, 11 novel regulators were identified using drosophila and zebrafish. This study validates zebrafish as a reliable platform for identifying the function of mutated genes in human disease.

Chemical screens and drug discovery. Zebrafish are amenable to largescale drug screens because the larvae can be exposed to drugs by simply placing them in a 96-well plate and adding chemicals to the water, though high-throughput chemical screens are less feasible in adult fish due to their larger size. To increase the scale of embryo collection, a specialized breeding tank was designed, allowing the collection of thousands of embryos that are developmentally synchronized (94). Several large chemical screens have been performed in the past 5 years (95) that have identified important new compounds that are currently being tested in clinical trials $(96,97)$. In a screen aimed at discovering novel regulators of hematopoietic stem cell formation, North et al. found that prostaglandin E2 is an important and conserved regulator of hematopoietic stem cell number (96) that interacts with the Wnt signaling pathway to regulate HSC regeneration (98). These results have been confirmed in vitro and in vivo in a murine model, and prostaglandins are currently being tested in a clinical trial to potentially enhance hematopoietic stem engraftment after bone marrow depletion $(96,99)$. From a large chemical screen for neural crest regulators, White et al. found that leflunomide, a drug that it is used to treat rheumatoid arthritis, affects transcriptional elongation of genes that are required for neural crest development and melanoma growth (97). Leflunomide is also currently being tested in melanoma patients in combination with a BRAF inhibitor. Both of these studies validate the feasibility of discovering relevant compounds using zebrafish combined with successful translation into the clinic. 
Transplantation of tissues. Cell and tissue transplantation techniques have been used in zebrafish embryos to investigate the state of cell commitment, inductive interactions between embryonic tissues, and defective tissues in mutant embryos $(100,101)$. More recently, the zebrafish system has emerged as reliable model for tumor transplantation assays $(102,103)$. Cancer cell lines derived from different species and tissues have been transplanted into zebrafish in order to study tumor cell reprogramming (104), migration, metastasis, and effects on vasculogenesis (102, 104-108). The generation of the double pigmentation mutant casper $(109,110)$, which has a completely transparent body even in adulthood, has greatly facilitated noninvasive tracing of tumor cells using fluorescent imaging techniques (111), making zebrafish a highly attractive transplantation model. Several laboratories have transplanted human tumors into zebrafish embryos and can examine aspects of tumor biology such as angiogenesis (112). Marrow transplantation has also been done in the zebrafish system, opening the door for solid organ transplants in the future.

\section{Concluding remarks}

Fish and humans share a high similarity of gene function involved in physiological processes. Modeling disease in the zebrafish provides a convenient and powerful platform in which genetic or chemical suppressors or enhancers of the disease phenotype can be identified and studied. Zebrafish will be beneficial for the understanding of human disease pathogenesis and ultimately the discovery of new therapeutic agents for a wide range of disorders.

\section{Acknowledgments}

We are grateful to Ellen van Rooijen for critical reading of the manuscript and discussion. We thank Charles Kaufman and Richard Mark White for critical reading of the manuscript. L.I. Zon is an investigator of the Howard Hughes Medical Institute.

Address correspondence to: Leonard I. Zon, Children's Hospital Boston, Karp Family Research Building, 300 Longwood Avenue, Boston, Massachusetts 02115, USA. Phone: 617.919.2069; Fax: 617.730.0222; E-mail: zon@enders.tch.harvard.edu.
1. Langheinrich U. Zebrafish: a new model on the pharmaceutical catwalk. Bioessays. 2003;25(9):904-912.

2. Postlethwait J, Amores A, Cresko W, Singer A, Yan YL. Subfunction partitioning, the teleost radiation and the annotation of the human genome. Trends Genet. 2004;20(10):481-490.

3. Amsterdam A, Hopkins N. Mutagenesis strategies in zebrafish for identifying genes involved in development and disease. Trends Genet. 2006;22(9):473-478.

4. Haffter $\mathrm{P}$, et al. The identification of genes with unique and essential functions in the development of the zebrafish, Danio rerio. Development. 1996;123:1-36.

5. McGrail M, et al. Somatic mutagenesis with a Sleeping Beauty transposon system leads to solid tumor formation in zebrafish. PLoS One. 2011; 6(4):e18826

6. Lan CC, Leong IU, Lai D, Love DR. Disease modeling by gene targeting using microRNAs. Methods Cell Biol. 2011;105:419-436.

7. Meng X, Noyes MB, Zhu LJ, Lawson ND, Wolfe SA. Targeted gene inactivation in zebrafish using engineered zinc-finger nucleases. Nat Biotechnol. 2008; 26(6):695-701.

8. Wienholds E, Plasterk RH. Target-selected gene inactivation in zebrafish. Methods Cell Biol. 2004; 77:69-90.

9. Wienholds E, van Eeden F, Kosters M, Mudde J, Plasterk RH, Cuppen E. Efficient target-selected mutagenesis in zebrafish. Genome Res. 2003; 13(12):2700-2707

10. Stemple DL. TILLING--a high-throughput harvest for functional genomics. Nat Rev Genet. 2004; 5(2):145-150.

11. Berghmans S, et al. tp53 mutant zebrafish develop malignant peripheral nerve sheath tumors. Proc Natl Acad Sci U S A. 2005;102(2):407-412.

12. Lieber MR. The mechanism of double-strand DNA break repair by the nonhomologous DNA end-joining pathway. Annu Rev Biochem. 2010;79:181-211.

13. Urnov FD, Rebar EJ, Holmes MC, Zhang HS, Gregory PD. Genome editing with engineered zinc finger nucleases. Nat Rev Genet. 2011;11(9):636-646.

14. Zhu C, et al. Evaluation and application of modularly assembled zinc-finger nucleases in zebrafish. Development. 2011;138(20):4555-4564.

15. Zhu C, et al. Evaluation and application of modularly assembled zinc-finger nucleases in zebrafish. Development. 2011;138(20):4555-4564.

16. Li T, et al. Modularly assembled designer TAL effector nucleases for targeted gene knockout and gene replacement in eukaryotes. Nucleic Acids Res.
2011;39(14):6315-6325.

17. Cermak T, et al. Efficient design and assembly of custom TALEN and other TAL effector-based constructs for DNA targeting. Nucleic Acids Res. 2011; 39(12):e82.

18. Gupta A, Meng X, Zhu LJ, Lawson ND, Wolfe $\mathrm{SA}$. Zinc finger protein-dependent and -independent contributions to the in vivo off-target activity of zinc finger nucleases. Nucleic Acids Res. 2010; 39(1):381-392.

19. Kikuta H, Kawakami K. Transient and stable transgenesis using tol2 transposon vectors. Methods $\mathrm{Mol}$ Biol. 2009;546:69-84.

20. Suster ML, Kikuta H, Urasaki A, Asakawa K, Kawakami K. Transgenesis in zebrafish with the tol2 transposon system. Methods Mol Biol. 2009; 561:41-63.

21. Kawakami K, et al. zTrap: zebrafish gene trap and enhancer trap database. BMC Dev Biol. 2010;10:105.

22. Emelyanov A, Parinov S. Mifepristone-inducible LexPR system to drive and control gene expression in transgenic zebrafish. Dev Biol. 2008;320(1):113-121.

23. Mosimann C, Zon LI. Advanced zebrafish transgenesis with Tol2 and application for Cre/lox recombination experiments. Methods Cell Biol. 2011; 104:173-194.

24. Scott EK, et al. Targeting neural circuitry in zebrafish using GAL4 enhancer trapping. Nat Methods. 2007; 4(4):323-326

25. Patton EE, et al. BRAF mutations are sufficient to promote nevi formation and cooperate with p53 in the genesis of melanoma. Curr Biol. 2005; 15(3):249-254.

26. Santoriello C, et al. Kita driven expression of oncogenic HRAS leads to early onset and highly penetrant melanoma in zebrafish. PLoS One. 2010; 5(12):e15170.

27. Brownlie A, et al. Positional cloning of the zebrafish sauternes gene: a model for congenital sideroblastic anaemia. Nat Genet. 1998;20(3):244-250.

28. Donovan A, et al. Positional cloning of zebrafish ferroportin 1 identifies a conserved vertebrate iron exporter. Nature. 2000;403(6771):776-781.

29. Shafizadeh E, et al. Characterization of zebrafish merlot/chablis as non-mammalian vertebrate models for severe congenital anemia due to protein 4.1 deficiency. Development. 2002;129(18):4359-4370.

30. Taylor AM, Zon LI. Modeling Diamond Blackfan anemia in the zebrafish. Semin Hematol. 2011; 48(2):81-88.

31. van Rooijen E, et al. Zebrafish mutants in the von Hippel-Lindau tumor suppressor display a hypoxic response and recapitulate key aspects of Chuvash polycythemia. Blood. 2009;113(25):6449-6460.

32. Dooley KA, et al. montalcino, A zebrafish model for variegate porphyria. Exp Hematol. 2008; 36(9):1132-1142.

33. Montosi G, et al. Autosomal-dominant hemochromatosis is associated with a mutation in the ferroportin (SLC11A3) gene. J Clin Invest. 2001; 108(4):619-623.

34. Danilova N, Sakamoto KM, Lin S. Ribosomal protein S19 deficiency in zebrafish leads to developmental abnormalities and defective erythropoiesis through activation of $\mathrm{p} 53$ protein family. Blood. 2008;112(13):5228-5237.

35. Uechi T, Nakajima Y, Chakraborty A, Torihara H, Higa S, Kenmochi N. Deficiency of ribosomal protein S19 during early embryogenesis leads to reduction of erythrocytes in a zebrafish model of Diamond-Blackfan anemia. Hum Mol Genet. 2008; 17(20):3204-3211.

36. Tamary H, Alter BP. Current diagnosis of inherited bone marrow failure syndromes. Pediatr Hematol Oncol. 2007;24(2):87-99.

37. Taylor AM, Humphries JM, White RM, Murphey RD, Burns CE, Zon LI. Hematopoietic defects in rps29 mutant zebrafish depend upon p53 activation. Exp Hematol. 2012;40(3):228-237.

38. Linet MS, Ries LA, Smith MA, Tarone RE, Devesa SS. Cancer surveillance series: recent trends in childhood cancer incidence and mortality in the United States. J Natl Cancer Inst. 1999;91(12):1051-1058.

39. Langenau DM, et al. Myc-induced T cell leukemia in transgenic zebrafish. Science. 2003; 299(5608):887-890.

40. Feng H, et al. Heat-shock induction of T-cell lymphoma/leukaemia in conditional Cre/lox-regulated transgenic zebrafish. Br J Haematol. 2007; 138(2):169-175

41. Chen J, Jette C, Kanki JP, Aster JC, Look AT, Griffin JD. NOTCH1-induced T-cell leukemia in transgenic zebrafish. Lenkemia. 2007;21(3):462-471.

42. Feng $H$, et al. T-lymphoblastic lymphoma cells express high levels of BCL2, S1P1, and ICAM1, leading to a blockade of tumor cell intravasation. Cancer Cell. 2010;18(4):353-366.

43. Mizgirev IV, Revskoy S. A new zebrafish model for experimental leukemia therapy. Cancer Biol Ther. 2010;9(11):895-902.

44. Rudner LA, et al. Shared acquired genomic changes in zebrafish and human T-ALL. Oncogene. 2011;30(41):4289-4296.

45. Amatruda JF, Shepard JL, Stern HM, Zon LI. 
Zebrafish as a cancer model system. Cancer Cell. 2002;1(3):229-231.

46. Liu S, Leach SD. Zebrafish models for cancer. Annu Rev Pathol. 2011;6:71-93.

47. Davies H, et al. Mutations of the BRAF gene in human cancer. Nature. 2002;417(6892):949-954.

48. Patton EE, et al. BRAF mutations are sufficient to promote nevi formation and cooperate with p53 in the genesis of melanoma. Curr Biol. 2005; 15(3):249-254.

49. Avery-Kiejda KA, et al. P53 in human melanoma fails to regulate target genes associated with apoptosis and the cell cycle and may contribute to proliferation. BMC Cancer. 2011;11:203.

50. Santoriello C, et al. Kita driven expression of oncogenic HRAS leads to early onset and highly penetrant melanoma in zebrafish. PLoS One. 2010; 5(12):e15170.

51. Ceol CJ, et al. The histone methyltransferase SETDB1 is recurrently amplified in melanoma and accelerates its onset. Nature. 2011;471(7339):513-517.

52. Stainier DY, et al. Mutations affecting the formation and function of the cardiovascular system in the zebrafish embryo. Development. 1996;123:285-292.

53. Sehnert AJ, Huq A, Weinstein BM, Walker C, Fishman M, Stainier DY. Cardiac troponin T is essential in sarcomere assembly and cardiac contractility. Nat Genet. 2002;31(1):106-110.

54. Xu X, et al. Cardiomyopathy in zebrafish due to mutation in an alternatively spliced exon of titin. Nat Genet. 2002;30(2):205-209.

55. Knöll R, et al. Laminin-alpha4 and integrin-linked kinase mutations cause human cardiomyopathy via simultaneous defects in cardiomyocytes and endothelial cells. Circulation. 2007;116(5):515-525.

56. Milan DJ, et al. Drug-sensitized zebrafish screen identifies multiple genes, including GINS3, as regulators of myocardial repolarization. Circulation. 2009;120(7):553-559.

57. Macrae CA. Cardiac Arrhythmia: In vivo screening in the zebrafish to overcome complexity in drug discovery. Expert Opin Drug Discov. 2010;5(7):619-632.

58. Peal DS, et al. Novel chemical suppressors of long QT syndrome identified by an in vivo functional screen. Circulation. 2011;123(1):23-30.

59. Porrello ER, et al. Transient regenerative potential of the neonatal mouse heart. Science. 2011; 331(6020):1078-1080.

60. Poss KD, Wilson LG, Keating MT. Heart regeneration in zebrafish. Science. 2002;298(5601):2188-2190.

61. Chablais F, Veit J, Rainer G, Jazwinska A. The zebrafish heart regenerates after cryoinjuryinduced myocardial infarction. BMC Dev Biol. 2011;11:21.

62. Jopling C, Sleep E, Raya M, Marti M, Raya A, Izpisúa Belmonte JC. Zebrafish heart regeneration occurs by cardiomyocyte dedifferentiation and proliferation. Nature. 2010;464(7288):606-609.

63. Lepilina A, et al. A dynamic epicardial injury response supports progenitor cell activity during zebrafish heart regeneration. Cell. 2006;127(3):607-619.

64. Riley PR, Smart N. Thymosin beta4 induces epicardium-derived neovascularization in the adult heart. Biochem Soc Trans. 2009;37(pt 6):1218-1220.

65. Smart N, et al. De novo cardiomyocytes from within the activated adult heart after injury. Nature. 2011; 474(7353):640-644.

66. Bassett DI, Currie PD. The zebrafish as a model for muscular dystrophy and congenital myopathy. Hum Mol Genet. 2003;12 spec no 2:R265-R270.

67. Kawahara G, Karpf JA, Myers JA, Alexander MS, Guyon JR, Kunkel LM. Drug screening in a zebrafish model of Duchenne muscular dystrophy. Proc Natl Acad Sci U S A. 2011;108(13):5331-5336.

68. Asai A, Sahani N, Kaneki M, Ouchi Y, Martyn JA, Yasuhara SE. Primary role of functional ischemia, quantitative evidence for the two-hit mechanism, and phosphodiesterase-5 inhibitor therapy in mouse muscular dystrophy. PLoS One. 2007;2(8):e806.

69. Swanhart LM, Cosentino CC, Diep CQ, Davidson AJ, de Caestecker M, Hukriede NA. Zebrafish kidney development: basic science to translational research. Birth Defects Res C Embryo Today. 2011; 93(2):141-156.

70. Cao Y, Park A, Sun Z. Intraflagellar transport proteins are essential for cilia formation and for planar cell polarity. J Am Soc Nephrol. 2010;21(8):1326-1333.

71. Sun Z, Amsterdam A, Pazour GJ, Cole DG, Miller MS, Hopkins N. A genetic screen in zebrafish identifies cilia genes as a principal cause of cystic kidney. Development. 2004;131(16):4085-4093.

72. van Rooijen E, Giles RH, Voest EE, van Rooijen C, Schulte-Merker S, van Eeden FJ. LRRC50, a conserved ciliary protein implicated in polycystic kidney disease. J Am Soc Nephrol. 2008;19(6):1128-1138.

73. Tobin JL, Beales PL. Restoration of renal function in zebrafish models of ciliopathies. Pediatr Nephrol. 2008;23(11):2095-2099.

74. Diep CQ, et al. Identification of adult nephron progenitors capable of kidney regeneration in zebrafish. Nature. 2011;470(7332):95-100.

75. Brennan CH. Zebrafish behavioural assays of translational relevance for the study of psychiatric disease. Rev Neurosci. 2011;22(1):37-48.

76. Morris AC. The genetics of ocular disorders: insights from the zebrafish. Birth Defects Res C Embryo Today. 2011;93(3):215-228.

77. Newman M, Verdile G, Martins RN, Lardelli M. Zebrafish as a tool in Alzheimer's disease research. Biochim Biophys Acta. 2011;1812(3):346-352.

78. Xi Y, Noble S, Ekker M. Modeling neurodegeneration in zebrafish. Curr Neurol Neurosci Rep. 2011; 11(3):274-282.

79. Kokel D, et al. Rapid behavior-based identification of neuroactive small molecules in the zebrafish. Nat Chem Biol. 2010;6(3):231-237.

80. Rihel J, et al. Zebrafish behavioral profiling links drugs to biological targets and rest/wake regulation. Science. 2010;327(5963):348-351.

81. Bibliowicz J, Tittle RK, Gross JM. Toward a better understanding of human eye disease insights from the zebrafish, Danio rerio. Prog Mol Biol Transl Sci. 2011;100:287-330.

82. Lee J, Willer JR, Willer GB, Smith K, Gregg RG, Gross JM. Zebrafish blowout provides genetic evidence for Patched1-mediated negative regulation of Hedgehog signaling within the proximal optic vesicle of the vertebrate eye. Dev Biol. 2008; 319(1):10-22.

83. Moosajee M, Gregory-Evans K, Ellis CD, Seabra MC, Gregory-Evans CY. Translational bypass of nonsense mutations in zebrafish rep1, pax2.1 and lamb1 highlights a viable therapeutic option for untreatable genetic eye disease. Hum Mol Genet. 2008;17(24):3987-4000.

84. Neuhauss SC, et al. Genetic disorders of vision revealed by a behavioral screen of 400 essential loci in zebrafish. J Neurosci. 1999;19(19):8603-8615.

85. Lancaster MA, Gleeson JG. The primary cilium as a cellular signaling center: lessons from disease. Curr Opin Genet Dev. 2009;19(3):220-229.

86. Morris AC. The genetics of ocular disorders: insights from the zebrafish. Birth Defects Res C Embryo Today. 2011;93(3):215-228.

87. Ceol CJ, et al. The histone methyltransferase SETDB1 is recurrently amplified in melanoma and accelerates its onset. Nature. 2011;471(7339):513-517.

88. Norton N, et al. Genome-wide studies of copy number variation and exome sequencing identify rare variants in BAG3 as a cause of dilated cardiomyopathy. Am J Hum Genet. 2011;88(3):273-282.

89. Weterman MA, et al. A frameshift mutation in LRSAM1 is responsible for a dominant hereditary polyneuropathy. Hum Mol Genet. 2012;21(2):358-370.

90. Bem D, et al. Loss-of-function mutations in RAB18 cause Warburg micro syndrome. Am J Hum Genet.
2011;88(4):499-507

91. Jiao S, et al. The conserved clusterin gene is expressed in the developing choroid plexus under the regulation of notch but not IGF signaling in zebrafish. Endocrinology. 2011;152(5):1860-1871.

92. O'Toole JF, et al. Individuals with mutations in XPNPEP3, which encodes a mitochondrial protein, develop a nephronophthisis-like nephropathy. J Clin Invest. 2010;120(3):791-802.

93. Gieger C, et al. New gene functions in megakaryopoiesis and platelet formation. Nature. 2011; 480(7376):201-208.

94. Adatto I, Lawrence C, Thompson M, Zon LI. A new system for the rapid collection of large numbers of developmentally staged zebrafish embryos. PLoS One. 2011;6(6):e21715.

95. Tan JL, Zon LI. Chemical screening in zebrafish for novel biological and therapeutic discovery. Methods Cell Biol. 2011;105:493-516.

96. North TE, et al. Prostaglandin E2 regulates vertebrate haematopoietic stem cell homeostasis. Nature. 2007;447(7147):1007-1011.

97. White RM, et al. DHODH modulates transcriptional elongation in the neural crest and melanoma. Nature. 2011;471(7339):518-522.

98. Goessling W, et al. Genetic interaction of PGE2 and Wnt signaling regulates developmental specification of stem cells and regeneration. Cell. 2009;136(6):1136-1147

99. Goessling W, et al. Prostaglandin E2 enhances human cord blood stem cell xenotransplants and shows long-term safety in preclinical nonhuman primate transplant models. Cell Stem Cell. 2011; 8(4):445-458.

100.Mizuno T, Shinya M, Takeda H. Cell and tissue transplantation in zebrafish embryos. Methods Mol Biol. 1999;127:15-28.

101.Xu Q, Stemple D, Joubin K. Microinjection and cell transplantation in zebrafish embryos. Methods Mol Biol. 2008;461:513-520.

102. Stoletov K, Montel V, Lester RD, Gonias SL, Klemke R. High-resolution imaging of the dynamic tumor cell vascular interface in transparent zebrafish. Proc Natl Acad Sci U S A. 2007;104(44):17406-17411.

103. Taylor AM, Zon LI. Zebrafish tumor assays: the state of transplantation. Zebrafish. 2009;6(4):339-346.

104.Diez-Torre A, et al. Reprogramming of melanoma cells by embryonic microenvironments. Int J Dev Biol. 2009;53(8-10):1563-1568.

105. Haldi M, Ton C, Seng WL, McGrath P. Human melanoma cells transplanted into zebrafish proliferate, migrate, produce melanin, form masses and stimulate angiogenesis in zebrafish. Angiogenesis. 2006;9(3):139-151.

106. Lee LM, Seftor EA, Bonde G, Cornell RA, Hendrix MJ. The fate of human malignant melanoma cells transplanted into zebrafish embryos: assessment of migration and cell division in the absence of tumor formation. Dev Dyn. 2005;233(4):1560-1570.

107. Nicoli S, Ribatti D, Cotelli F, Presta M. Mammalian tumor xenografts induce neovascularization in zebrafish embryos. Cancer Res. 2007;67(7):2927-2931.

108. Topczewska JM, et al. Embryonic and tumorigenic pathways converge via Nodal signaling: role in melanoma aggressiveness. Nat Med. 2006;12(8):925-932.

109. Wenner M. The most transparent research. Nat Med. 2009;15(10):1106-1109.

110. White RM, et al. Transparent adult zebrafish as a tool for in vivo transplantation analysis. Cell Stem Cell. 2008;2(2):183-189.

111.Ignatius MS, Langenau DM. Fluorescent imaging of cancer in zebrafish. Methods Cell Biol. 2011; 105:437-459.

112.Stoletov K, Montel V, Lester RD, Gonias SL, Klemke R. High-resolution imaging of the dynamic tumor cell vascular interface in transparent zebrafish. Proc Natl Acad Sci U S A. 2007; 104(44):17406-17411. 